\title{
Optimization of Network Signal Timing Based on Dynamic Traffic Guidance
}

\author{
Yanlong Chu \\ Police Administration Department, Liaoning Police College, Dalian City, Liaoning Province, 116036, China
}

Keywords: dynamic traffic guidance, network signal timing, optimization

\begin{abstract}
Under the condition of dynamic traffic guidance, the path selection behavior of divers would results in the new road network traffic condition, and then the influence of traffic flow on network signal timing optimization must be taken into account in the process of matching. This paper presented the scheme of network signal timing, and then the algorithm of integer programming and mountain climbing was used to optimize the network signal timing in dynamic traffic guidance. At each research point, the path equalization was carried out to make the calculated target function - the road network performance index. At last, this algorithm was used to calculate the road network instance, and the results showed that the proposed algorithm could optimize the scheme of equalization network signal timing.
\end{abstract}

\section{Introduction}

In the existing regional traffic signal control system, whether SCOOT or SCATS, should be able to obtain the number of vehicles coming through the intersection in all directions, the arrival rate of traffic flow at each intersection. In the absence of a dynamic traffic guidance system, due to drivers have no significance on the road condition, most of the drivers would not choose a new path, so that traffic flows in this way are relatively stable, and there is a more successful optimization method in the case of fixed network traffic. With the development and application of ITS technology, the availability of advanced road traffic guidance information has caused random alternations in the drivers' selection behavior of path. Thus, the traffic of road traffics are changed, and the arrival rate of traffic flow at each intersection is determined by the route selection of drivers under the guidance strategy. Corresponding to the new road network traffic, the signal control system must take into account the negative impact of the heavy new distribution of traffic on road networks, otherwise the proposed scheme would not be optimal.

\section{The Scheme of Network Signal Timing}

The research of this paper is how to optimize the scheme of network signal timing, so it is important to consider how to express the scheme of network signal timing. In general, the expression of the timing parameters of signal intersection adopted the "phase" and "stage" methods, which is more complicated for multi-phase signal matching. In addition, the research object in this paper is the scheme of urban road networks. In the urban road networks, the distance between adjacent intersections is shorter, and the coordination control of adjacent intersections could get good results. Therefore, the phase offset between adjacent intersections must be considered.

The method of signal group was used to describe the parameters of road network distribution in this paper. Considering the set of $\mathrm{A}$ in the road networks, and $\mathrm{N}$ in the set of nodes, and assuming the periodic time of all nodes is $\mathrm{c}$ in the TRANSYT mode. The right of passage in specific signal groups are represented by $\theta \mathrm{mn}$, and $\Phi \mathrm{mn}$, which represent the starting time and duration of green light at the intersection $\mathrm{m}$ and signal group $\mathrm{n}$. In order to describe the coordination control of adjacent intersections, a master clock is defined as the absolute time reference for all intersections. According to the difference between the starting time of green light and the master clock, the $\theta \mathrm{mn}$ could be obtained. In addition, the NGm is the set of $\mathrm{m}$ signal group. According to the above parameters, combining with the constraint condition, the feasible regions could be solved.

The periodic time: if the periodic time should be optimized, the following feasible region should 
be defined.

$$
c_{\min } \leq c \leq c_{\max }
$$

Here, $C_{\min }$ and $C_{\max }$ means the maximum and minimum of periodic time.

The duration of green light: the minimum constraints of green light are given for safety reasons. For node $\mathrm{m}$ and signal group $\mathrm{n}$.

$$
\zeta_{\min } \leq \phi \leq c
$$

Here, $\zeta_{\min }$ is the minimum time of green light for node $m$ and signal group $n$.

The change interval of green time: for conflicting signal groups in each intersections, it is necessary to give the maximum interval of the green light, so that the vehicle in the next signal group could reach the impact point by the following signal group. For node $m$, the conflicting signal groups could be described as below.

$$
\theta_{m n}+\phi_{m n}+\kappa_{m}(n, l) \leq \theta_{m l}+c \Omega_{m}(n, l)
$$

Here, $\kappa_{m}(n, l)$ represents the interval between the finishing time of signal group $\mathrm{n}$ and the starting time of signal group $1 ; \Omega_{m}(n, l)$ is a correlation index between 0 and 1 . As shown in figure 1, there are 8 signal groups and 5 nodes (each line corresponds to a signal group, line 27 corresponds to signal group 1, which is arranged in a sequence, and line 34 corresponds to signal group 8). In addition, the timing parameters in each signal group are shown in Figure 2.

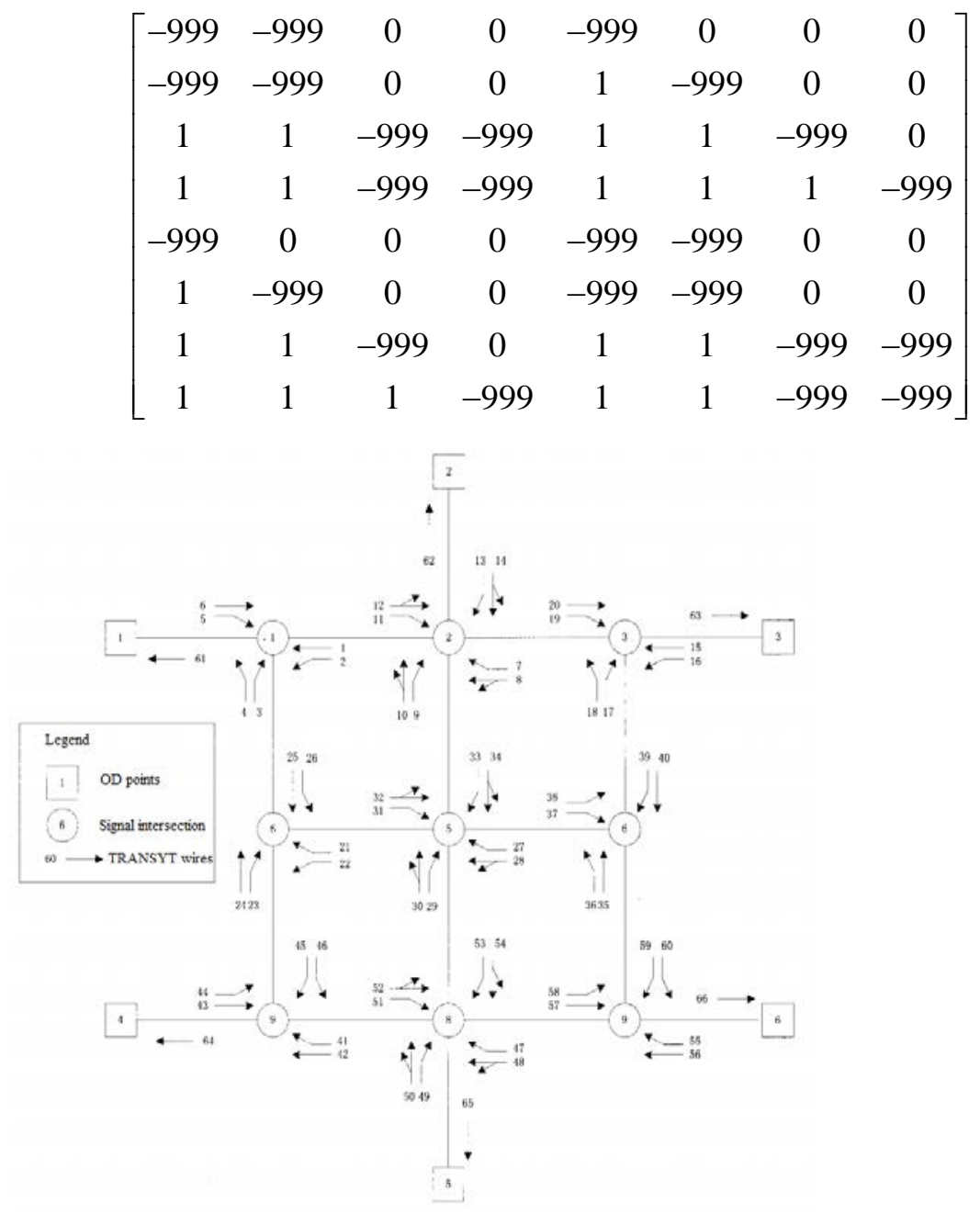

Figure 1 the road networks 
As shown in Figure 2, the starting time of green light in group 1 is 0 , and its duration is 20s. Therefore, the following matrix of correlation index could be obtained. The -999 means there is no conflicts between signal groups. According to the scheme of timing, the starting time of signal group 1 and 5 is 0 , so that the row 1 and row 5 in the correlation index matrix are -999 and 0 . On the contrary, the starting time of signal group 4 and 8 is 85s, so that the row 4 and row 8 in the correlation index matrix are -999 and 1.

Traffic capacity: To avoid oversaturation, a maximum acceptable satiety could be defined to make a connection flow on a certain crowding level. For line a, suppose that the upper traffic flow is controlled by intersection $\mathrm{m}$ and signal group $\mathrm{n}$, then:

$$
c v_{a} \leq \sigma_{a}\left(\phi_{m n}+\omega_{e}-\omega_{s}\right) s_{a}
$$

Here, $v_{a}, \sigma_{a}$ and $S_{a}$ represent the wire flow, acceptable saturation flow and saturation flow. $\omega_{e}$ and $\omega_{s}$ stand for the initial loss of green light and the end compensation of green light.

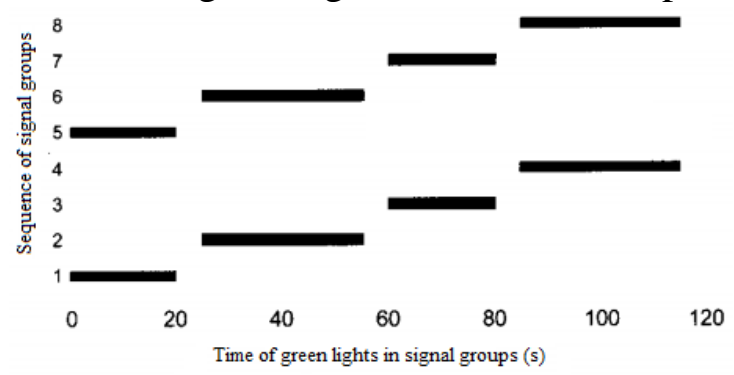

Figure 2 the scheme of timing of node 5 in road networks

\section{Case Study}

Considering the road network in Figure 1, there are 9 nodes, 66 wires and 6 OD points. The requirement of $\mathrm{OD}$ is displayed as below.

$$
\left[\begin{array}{cccccc}
9 & 91 & 125 & 223 & 9 & 8 \\
145 & 0 & 234 & 37 & 277 & 281 \\
273 & 178 & 0 & 29 & 124 & 132 \\
279 & 189 & 87 & 0 & 148 & 162 \\
163 & 78 & 131 & 247 & 0 & 69 \\
225 & 145 & 52 & 173 & 132 & 0
\end{array}\right] \text { veh } / \mathrm{hr}
$$

Under two different conditions, the fixed period and indefinite period are optimized and calculated. The results are shown in Figure 3. In the case of fixed period, the joint periodic time of intersection is 120s. On the other hand, the maximum periodic time of intersection is set in 120 in the indefinite period. After the optimization, the common periodic time is 102s. The performance indexes of road networks in two distinct cases are decreased by $44.3 \%$ and $4.54 \%$. In addition, Figure 3 also shows that the performance index of road networks has decreased significantly.

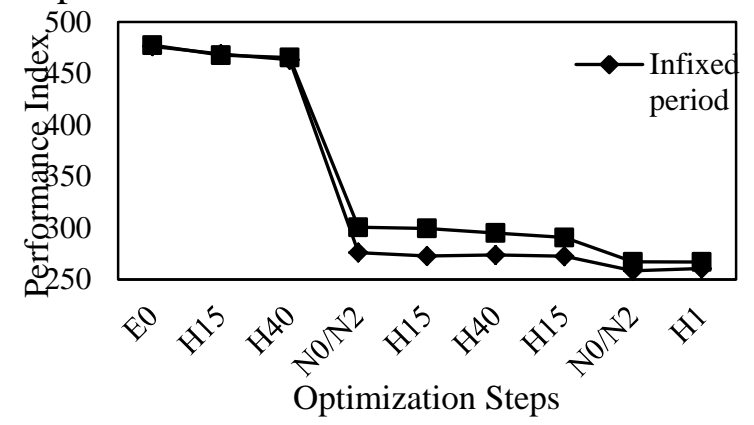

Figure 3 the optimization results based on two distinct methods 
In order to verify the effectiveness of the proposed optimization in this paper, 10 OD matrix are adopted to optimize the network signal timing. The elements in this matrix is random from 0 to 300 , and the elements on the diagonal are 0 . In the two different conditions, the optimization are calculated under fixed period and indefinite period, which results could be seen in Table 1.

Table 1 The optimization results under random OD requirements

\begin{tabular}{|c|c|c|c|c|c|c|}
\hline \multirow{3}{*}{ OD matrix } & \multicolumn{6}{|c|}{ Performance index } \\
\hline & \multirow[b]{2}{*}{ Initial value } & \multicolumn{2}{|c|}{ Fixed period time (120s) } & \multicolumn{3}{|c|}{ Infixed period time } \\
\hline & & T/veh & $\begin{array}{c}\text { Decreased } \\
\text { persentage/\% }\end{array}$ & $\begin{array}{c}\text { Optimization } \\
\text { time/s }\end{array}$ & T/veh & $\begin{array}{c}\text { Decreased } \\
\text { persentage/\% }\end{array}$ \\
\hline 1 & 478 & 266 & 44.3 & 102 & 261 & 45.4 \\
\hline 2 & 296 & 192 & 35.2 & 98 & 181 & 38.8 \\
\hline 3 & 397 & 270 & 32.0 & 96 & 247 & 37.6 \\
\hline 4 & 275 & 201 & 26.9 & 106 & 195 & 29.0 \\
\hline 5 & 319 & 215 & 32.7 & 100 & 191 & 40.1 \\
\hline 6 & 347 & 287 & 17.4 & 108 & 274 & 21.1 \\
\hline 7 & 611 & 361 & 40.9 & 120 & 362 & 40.7 \\
\hline 8 & 363 & 267 & 26.3 & 106 & 247 & 31.8 \\
\hline 9 & 369 & 242 & 34.5 & 110 & 242 & 34.5 \\
\hline 10 & 300 & 218 & 27.2 & 110 & 213 & 28.9 \\
\hline Mean value & 375 & 252 & 31.7 & - & 241 & 34.8 \\
\hline
\end{tabular}

\section{Conclusion}

Although there are real-time control system in our country, none of these systems consider the impact of traffic guidance system. The signal control mode and algorithm based on traffic guidance play an important role in the realization of real-time adaptive control system. Based on TRANSYT software, the traffic control algorithm was proposed under the traffic guidance conditions. The results showed the feasibility of traffic control optimization algorithm and the certain practical values.

\section{References}

[1] Luo Zhijun. Intelligent Management of Modern Urban Traffic--The Prqactices of Intelligent Traffic Management in Nanjing [J]. Journal of Industrial Engineering and Engineering Management, 2003, 17(2): 50-53.

[2] Ma Shoufeng, Li Yanjun, He Guoguang. System analysis for coordination modes between urban traffic control and vehicle route guidance [J]. Journal of Management sciences in China, 2003, 6(3): 71-78.

[3] Yang Dongkai, Wu Jinpei, Zhang Qishan. Intelligent Transport System and Its Informatics Model [J]. Journal of Beijing University of Aeronautics and Astronautics, 26(3): 270-273.

[4] Li Ruimin, Shi Qixin. Research on Integration of Urban Traffic Control and Route Guidance Based on Multi-agent [J]. Journal of Highway and Transportation Research and Development, 2004, 21(5): 109-112.

[5] Ji Xiaofeng, Huang Yongzhong, He Zenghui. Traffic state information extraction methods for traffic guidance [J]. Computer Engineering and Application, 2010, 46(25): 16-18.

[6] Xia Jin, Guo Hongwei. The General Situation and Development Trend of Urban Intelligence Transportation System Both at Home and Abroad [J]. Science \& Technology Progress and Policy, 2003, 20(1): 176-179. 